\title{
Terrain-adaptive infrared line-scan coding: A new image segmentation scheme
}

\author{
by D. Milovanovic * A. Marincic * and B. Wiecek ** \\ ${ }^{*}$ Faculty of Electrical Engineering, University of Belgrade, Serbia and Montenegro \\ ${ }^{* *}$ Technical University of Lodz, Institute of Electronics, Poland
}

\begin{abstract}
IR remote sensing and reconnaissance applications require transmission and/or store of line-scan thermal (IRLS) images in real-time. IRLS images exhibit a high degree of spatial redundancy that a codec reduces optimizing trade-off between bitrate and image distortion. In this paper, we propose adaptive coding based on joint optimization of image segmentation scheme and operational codec parameters. In computer implementation, we achieved significant coding gain using a new image segmentation scheme and advanced JPEG2000 codec.
\end{abstract}

\section{Introduction}

IR remote sensing and reconnaissance systems are constrained in terms of downlink communication bandwidth. To alleviate this limitation, the IR data must be compressed. Line-scan thermal (IRLS) images exhibit a high degree of spatial redundancy that must be properly exploited in any compression scheme. In addition, the DWT (Discrete Wavelet Transform) based JPEG2000 compression technology provide a range of fidelity options for the reconstructed data tailored to the end-use. This includes quick-look and browse, which exploits human visual perception deficiencies and offers the opportunity for high compression ratios; and machine exploitation, where visually-lossless coding is appropriate. In both the compression standards and the algorithms reported in the literature, transform coding has become the dominant approach for efficient image compression [1].

The efficiency of a transform encoder is determined both by the characteristics of the input IR data and by the capability of the coding algorithm to explore these characteristics. As a prerequisites for efficient coding of IRLS images, in our previous papers we studied statistical properties of IRLS images based on overall modeling of line-scanning process $[2,3,4]$ as well as image properties in wavelet transform domain [5]. In the next step, we systematically compared advanced transform-based image coders in order to find efficient operating parameters [6]. And finally, we proposed adaptive IRLS image coding based on DWT transform and spatial image segmentation along the line-scan direction [7]. In this paper, we propose further coding performance improvement based on terrain-adaptive segmentation along the flight direction and optimized JPEG2000 encoding parameters.

\section{IRLS image $D-R$ properties in line-scan and flight direction}

In transform coding of IRLS images, the two most important factors are the coding bit rate and picture quality. The coding bit rate $R$ determines the channel bandwidth required to transfer the coded data. One direct and widely used measure for the picture quality is the mean-square error (MSE) between the coded image and the original one. The reconstruction error introduced by compression is denoted by $D$. 
In our previous papers [7], we focuses on the optimal trade-off between average bit rate $R$ and average distortion $D$. However, it is well known that the $R-D$ behaviour of an image encoder is determined both by the characteristics of the input IRLS image and by the capability of the coding algorithm to explore these characteristics. Based on observed statistical properties of IRLS non-overlapping segments along the linescan and flight direction, we estimated $D-R$ operational functions of image segments in independent JPEG2000 encoding (Figure 1). For the constant bite rate $R$, it is observed variation of distortion $D$ in function of segment number. This is our motivation for decreasing average distortion of an image by adaptive segmentation and optimised bit rate allocation among segments.
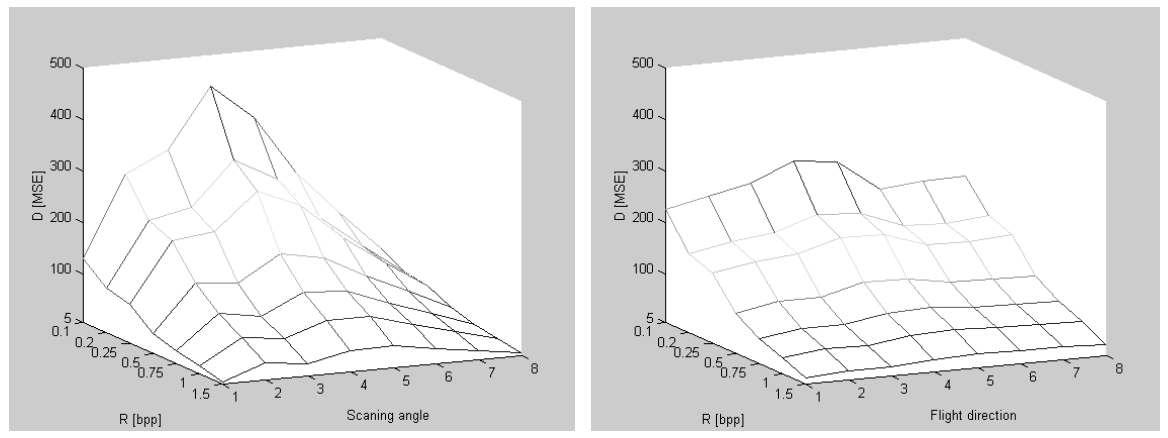

Fig. 1. Operational D-R functions of IRLS200 test image in independent JPEG2000 encoding of non-overlapped equal-size spatial image segments:

a) along the line-scan direction, b) along the flight direction.

\section{A new image adaptive segmentation scheme}

Our adaptive image segmentation is based on hierarchical data structures (Figure 2). A BT is a scheme of image segmentation based on a simple binary-tree structure. The primary characteristic of BT is that each node of the tree has 2 children which correspond to segments which area is 2 times smaller than parent node. Splitting original image (rote node) at the first top-level of the tree, 2 children node is generated. Recursively repeating segmentation on children nodes, the complete tree is generated in top-down fashion up to the pre-defined depth (level $L$ ). A QT is a scheme based on quad-tree structure where each node of the tree has 4 children [9].
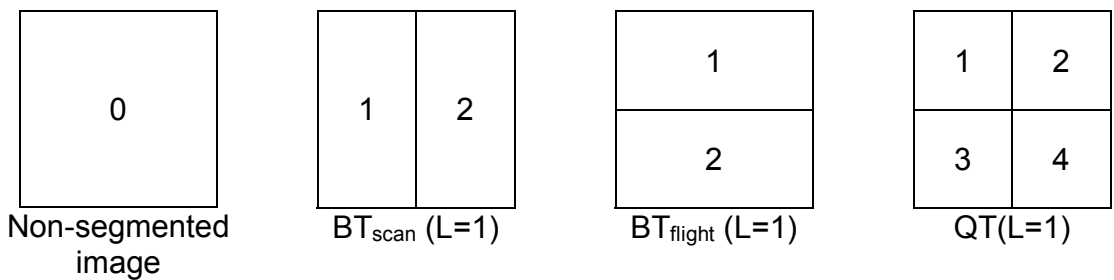

Fig. 2. Hierarchical IRLS image splitting in different tree-structures: non-segmented parent image and children segments in $\mathbf{B T}_{\text {scan }}$ binary-tree along the scan-line direction, $\mathbf{B T}_{\text {flight }}$ binary-tree along the flight direction and QT quad-tree. 
A factor that contributes to success of adaptive segmentation and coding of IRLS image is the choice of the parent node splitting decision criterion. A good criterion should determine whether or not segment should be merged or split to minimize average distortion $D$ for given bit rate $R$. In our work we propose Lagrangian unconstrained cost function $J(D, R, \lambda)$ of the mean distortion $D$ and the rate $R$ scaled by a value $\lambda$. To find joint optimal segmentation and minimize $D$ for pre-defined constant $R$, the per-segment cost functions for parent and children are compared: if the sum of children costs are less than cost of the parent, split is performed. Also, the fast optimisation procedure based on bottom-up recursive $\mathrm{BT}_{\text {scan }}$ split/merge algorithm and convex iterative $\lambda$ search is proposed [7].

Further improvement of IRLS coding gain could be obtained by exploiting terrainadaptive $\mathrm{BT}_{\text {flight }}$ segmentation in the flight direction. The new segmentation scheme is based on dual $\mathrm{BT}_{\text {scan/fight }}$ binary-tree structure (Figure 3 ). The additional criterion is comparison of $\mathrm{BT}_{\text {scan }}$ and $\mathrm{BT}_{\text {flight }}$ cost functions in the node and split if the condition (1) is satisfied.

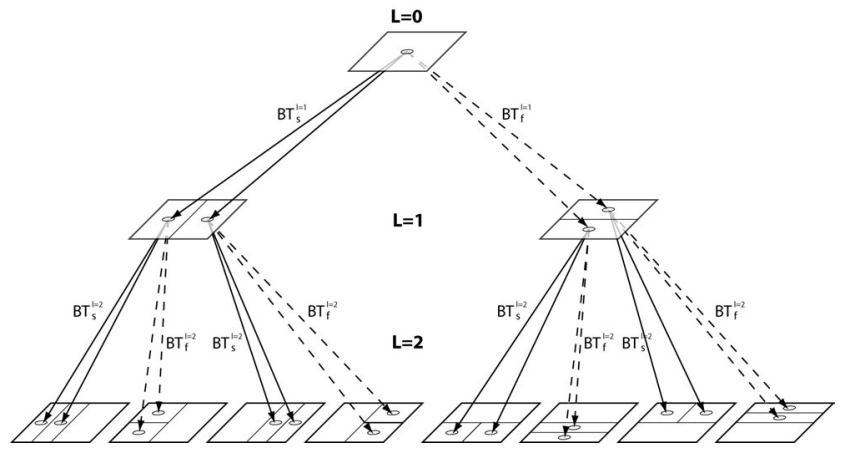

$$
\min \left\{J_{B T_{s}}\left(D_{l+1}, R_{l+1}, \lambda\right), J_{B T_{f}}\left(D_{l+1}, R_{l+1}, \lambda\right)\right\}<J\left(D_{l}, R_{l}, \lambda\right), \quad R=\sum_{l} R_{l}=\text { const }
$$
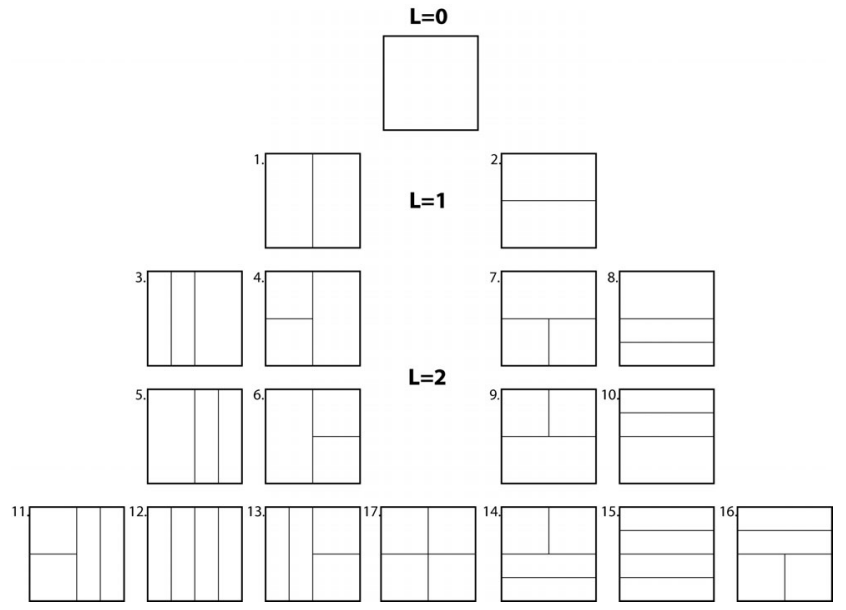

Fig. 3. Joint image segmentation and $\mathrm{D}-\mathrm{R}$ optimization procedure:

a) the new dual $\mathrm{BT}_{\text {scan/flight }}$ tree $(L=2)$, and

b) corresponding set of all possible image segmentation schemes in bottom-up split/merge procedure ( $L=2)$. 


\section{Coding gain results}

In computer implementation, we used JPEG2000 codec [8] in independent coding of segments and proposed optimisation algorithms. IRLS test images $(512 \times 512$ pixels, $8 \mathrm{bpp})$ are segmented hierarchically using different tree-structures in joint optimisation:

a) $\mathbf{B T}_{\text {scan }}$ binary-tree ( $\left.\mathrm{L}=3\right), \mathrm{N}=15$ different segmentation schemes are compared.

b) $\mathrm{BT}_{\text {flight }}$ binary-tree $(\mathrm{L}=3), \mathrm{N}=15$ different segmentation schemes are compared.

c) $\mathbf{B T}_{\text {scan/flight }}$ dual-tree $(\mathrm{L}=2), \mathrm{N}=16$ different segmentation schemes are compared.

d) QT quad-tree ( $\mathrm{L}=2), \mathrm{N}=16$ different segmentation schemes are compared.

Operational rate-distortion $D-R$ curves of each segment are computed applying JPEG2000 encoder with varying bit rates in the range 0.2-1 bpp (Compression Ratio 32-8) and measuring MSE of decoded image. For each type of segmentation tree and chosen bit rate $R$, we calculated optimum image segmentation and bit-allocation which gives minimum distortion $D$ (maximum PSNR). PSNR improvements over coding non-segmented IRLS200 image for fixed bit rate $R=0.75 \mathrm{bpp}$ are shown in Table 1. Decoded IRLS200 test images and adaptive segmentation schemes with optimum bit-allocation and minimum distortions are shown in Figure 4.

Table 1. PSNR coding gain of JPEG2000 coding of IRLS200 test image by adaptive segmentation and optimal bit rate allocation among segments.

\begin{tabular}{|c|c|c|c|c|c|}
\hline $\mathrm{R}=0.75 \mathrm{bpp}$ & Non-segmented & $\mathrm{BT}_{\text {scan }}(\mathrm{L}=3)$ & $\mathrm{BT}_{\text {flight }}(\mathrm{L}=3)$ & $\mathrm{BT}_{\text {scan/fight }}(\mathrm{L}=2)$ & $\mathrm{QT}(\mathrm{L}=2)$ \\
\hline $\mathrm{PSNR}[\mathrm{dB}]$ & 28.34 & 29.83 & 29.28 & 29.94 & 29.89 \\
\hline
\end{tabular}

It is observed significant adaptive coding gain over non-segmented IRLS test image. The new proposed $\mathrm{BT}_{\text {scan/flight }}$ dual-tree segmentation along the scan and flight direction out-perform single-tree segmentation $\mathrm{BT}_{\text {scan }}$ along the scan and $\mathrm{BT}_{\text {flight }}$ flight direction. Efficiency of the new scheme is very close to the more complex QT quadtree segmentation. Further advantage of proposed $\mathrm{BT}_{\text {scan/flight }}$ scheme is ability to explicit exploitation of IRLS systematic behaviour in the scan direction and terrain adaptation in the flight direction. It is observed that coding gain of adaptive segmentation in the scan direction should be greater at low bit rates (Figure 1) where visual quality of decoded IRLS image is decreasing.

Our future work is further improvement of IRLS coding efficiency by decreasing computational complexity of proposed optimisation algorithms: the $D-R$ optimisation process will perform only on those image regions of special interest; adaptive distortion $D$ weighting of segments and sub-optimal top-down optimisation as well as $D-R$ operational functions estimation and modelling based on statistical properties of segments. 
a) $\mathrm{BT}_{\text {scan }}$
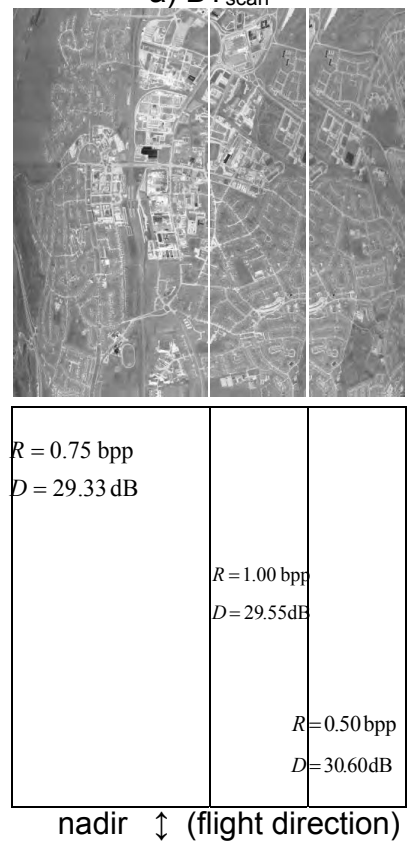

c) $\mathrm{BT}_{\text {scan/flight }}$

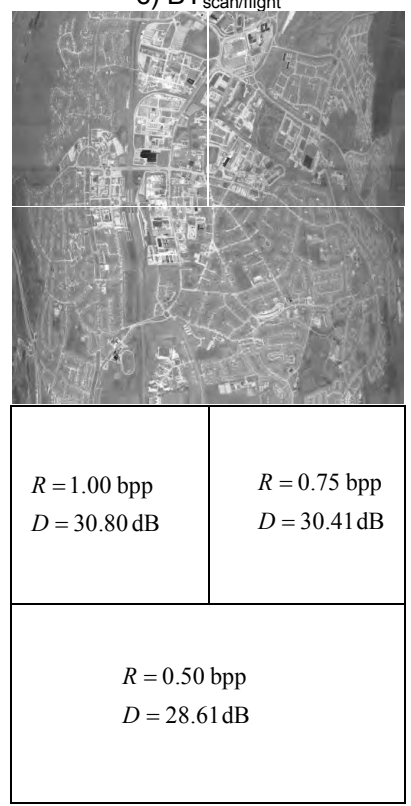

b) $\mathrm{BT}_{\text {flight }}$

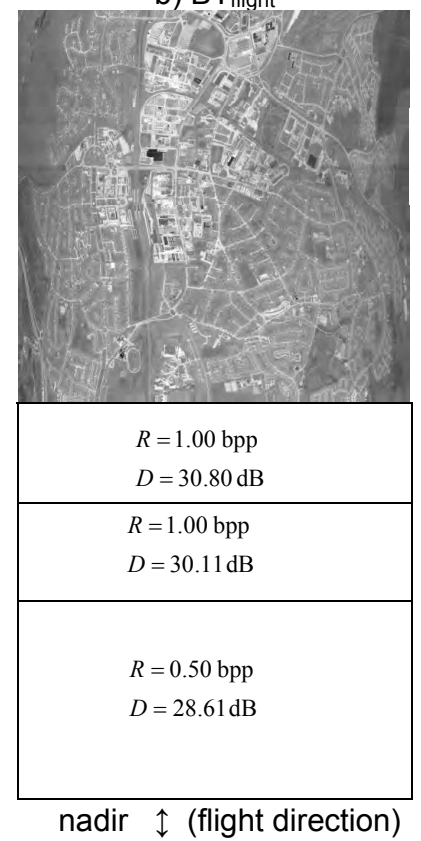

d) QT

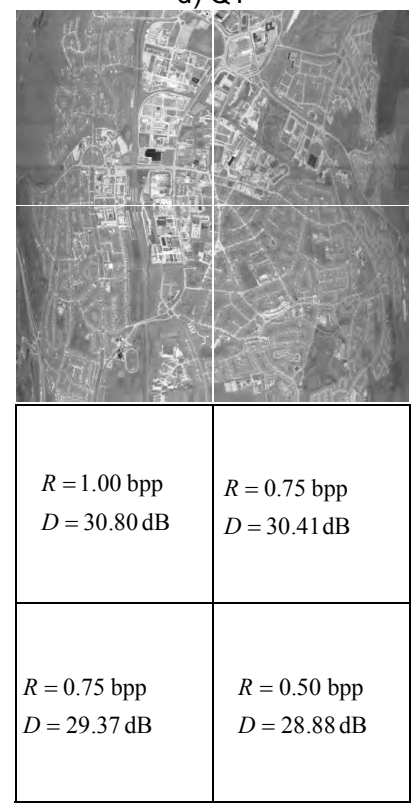

Fig. 4. Adaptive JPEG2000 ( $R=0.75 \mathrm{bpp})$ coding of IRLS200 test image based on joint optimisation of spatial segmentation and bit allocation among segments: a) $\mathrm{BT}_{\text {scan }}(\mathrm{L}=3)$ along the scan direction $(P S N R=29.83 \mathrm{~dB})$, b) $B T_{\text {flight }}(\mathrm{L}=3)$ along the flight direction $(P S N R=29.28 \mathrm{~dB}), c) \mathrm{BT}_{\text {scan/flight }}(\mathrm{L}=2)$ scan/flight direction $(P S N R=29.94 \mathrm{~dB})$, d) QT quad tree $(L=2)(P S N R=29.89 \mathrm{~dB})$. 


\section{REFERENCES}

[1] Saghri, J.; Tescher, A. and Reagan. J. Practical transform coding of multispectral imagery, IEEE Signal processing Magazine, January 1995.

[2] Barbarić, Ž.; Marinčić, A.; Petrović, G. and Milovanović. D. Thermal images generated by a line-scanning technique: statistical properties, Journal Applied Optics: Information Processing, vol.33 no.20 pp.4416-4419, 1994.

[3] Barbarić, Ž.; Marinčić, A.; Petrović, G. and Milovanović. D. Thermal-image generation by line-scanning technique: a new computer model", Journal Applied Optics: Information Processing, vol. 33, no.14, pp.2883-2890, 1994.

[4] Milovanović, D.; Marinčić, A.; Barbarić, Ž. and Petrović. G. Statistical analysis of computer generated thermal images based on overall modeling of linescanning process, QIRT 1994, Sorrento, Italy, pp.13-18.

[5] Milovanović, D.; Wiecek, B.; Marinčić, A.; Barbarić, Ž. and Petrović. G. Statistical analysis techniques for aerial infrared images in wavelets transform domain, QIRT 1996, Stuttgart, Germany, pp.368-373.

[6] Milovanović, D.; Wiecek, B.; Marinčić, A.; Petrović, G. and Barbarić. Ž. A comparative study of advanced frequency-domain coding techniques in compression of infrared line-scan images, QIRT 1998, Lodz, Poland, pp.342348.

[7] Milovanović, D.; Marinčić, A.; Wiecek, B.; Petrović, G. and Barbarić. Ž. Efficient transform coding of infrared line-scan images based on spatial adaptivity, QIRT, Reims, France, 2000.

[8] Milovanović, D.; Wiecek, B.; Marinčić, A.; Barbarić, Ž. and Petrović. G. Standard-based infrared line-scan image coding: JPEG2000 vs. MPEG-4 VTC, QIRT, Dubrovnik, Croatia, 2002

[9] Sullivan, G. and Baker. R. Efficient quad-tree coding of image and video, IEEE Trans. Image processing, vol.3, no.4, pp.327-331, May 1994. 